\title{
Impact extractive fracture of jointed steel plates of a bolted joint
}

\author{
M. Daimaruya ${ }^{1}$, H. Fujiki ${ }^{1}$, and H. Ambarita ${ }^{2}$ \\ ${ }^{1}$ Muroran Institute of Technology, Graduate School of Engineering, 27-1 Mizumoto, Muroran, 050-8585, Japan \\ ${ }^{2}$ University of Sumatera Utara, Dept. of Mechanical Engineering, Jl. Almamater USU Medan 20155, Indonesia
}

\begin{abstract}
This study is concerned with the development of a fracture criterion for the impact fracture of jointed steel plates of a bolted joint used in a car body. For the accurate prediction of crash characteristics of car bodies by computer-aided engineering (CAE), it is also necessary to examine the behavior and fracture of jointed steel plates subjected to impact loads. Although the actual impact fracture of jointed steel plates of a bolted joint used in cars is complicated, for simplifying the problem it might be classified into the shear fracture and the extractive fracture of jointed steel plates. Attention is given to the extractive fracture of jointed steel plates in this study. The extractive behavior and fracture of three kinds of steel plates used for cars are examined in experiments and numerical simulations. The impact extraction test of steel plates jointed by a bolt is performed using the one-bar method, together with the static test. In order to understand the mechanism of extractive fracture process of jointed steel plates, numerical simulations by a FEM code LS-DYNA are also carried out. The obtained results suggest that a stress-based fracture criterion may be developed for the impact extractive fracture of jointed steel plates of a bolted joint used in a car body.
\end{abstract}

\section{Introduction}

In the automotive industry collision safety and reliability of cars under impact loading has become more important in recent years, together with lightening of car body for reduction of $\mathrm{CO}_{2}$ emission. For the accurate prediction of crash characteristics of car bodies by CAE (computeraided engineering), it is necessary to examine not only the strength and fracture of welded joints such as spot welds and tailored blank welds [1-5], but also those of jointed steel plates of a bolted joint subjected to impact loads. There has been much work on bolted joints used in machine elements, in which strength of bolts, loosening processes and tightening torque of bolted joints are mainly examined $[6,7]$. However, we can hardly find investigations regarding the behavior and fracture of jointed steel plates under impact loading.

This study is concerned with the development of a fracture criterion for the impact fracture of jointed steel plates of bolted joints used in car bodies. The object is the part of lap-bolted joints connecting the suspension frame and the bracket of the suspension frame rear mounting in a car. Although the actual fracture of jointed steel plates of bolted joints on car collision is complicated, for simplifying the problem, it might be classified into the shear fracture and the extractive fracture of the jointed steel plates. In the previous papers we reported the shear deformation and fracture of steel plates jointed by a bolt under impact loadings $[8,9]$. In this study attention is given to the extractive fracture of jointed steel plates. The impact extractive behavior and fracture of three kinds of steel plates used for car bodies are examined in experiments and numerical simulations. The impact extraction test of steel plates jointed by a bolt is performed using the modified one-bar method for impact test [10,11], as well as the static test by a universal testing machine INSTRON 5586. In order to understand the mechanism of extractive fracture process of the jointed steel plates, numerical simulations by a FEM code LS-DYNA are also carried out. It is expected from the present study that a stress-based fracture criterion is developed for the impact extractive fracture of jointed steel plates of a bolted joint used in a car body.

\section{Static extractive experiments}

\subsection{Testing steel plates and procedure}

Although the actual fracture of jointed steel plates of the bolted joint used in cars is complicated on car collision, for simplifying the problem it might be classified into the shear fracture and the extractive fracture of the jointed steel plates. The extractive deformation and fracture of steel plates jointed by a bolt are focused in this study.

First, the static test is performed to obtain the static characteristics of steel plate specimens jointed by a bolt. Three kinds of steel plates, i.e., common steel with the tensile strength of $270 \mathrm{MPa}$ class (CR270) and high tensile strength steel with that of $440 \mathrm{MPa}$ class (CR440) and $590 \mathrm{MPa}$ class (CR590) used for car bodies, are employed in both the static and impact tests.

Figure 1 shows the size and configuration of the testing steel plates with $1.2 \mathrm{~mm}$ in thickness. A hole for inserting a bolt is drilled in the center of the specimen steel plates. The diameters of the hole are of three sorts, i.e., $d=6$, 8 and $10 \mathrm{~mm}$. The specimen plates are fixed by the upper and lower steel plate jigs with 12 bolts arranged around, and which are extracted using a universal testing machine INSTRON 5586 in static tests.

Figure 2 illustrates the schematic of the assembly of a specimen plate with a bolt, nuts and jig plates. The specimen steel plate is jointed by three kinds of bolts M6, M8 and M10, respectively. The corresponding washers are $13.5,17.5$ and $21.0 \mathrm{~mm}$ in outside diameter. The extractive deformation area of specimen plates is controlled varying the diameter $D$ of a hole bored in the center of the upper jig steel plate of S45C with $25 \mathrm{~mm}$ in thickness. In static extractive tests five kinds of the upper plate jig with 


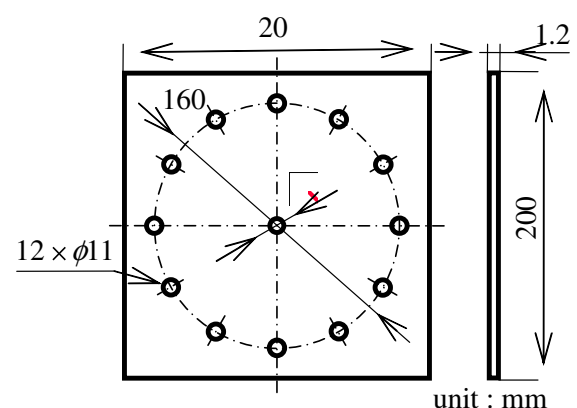

Fig. 1. Dimension of steel plate specimen.
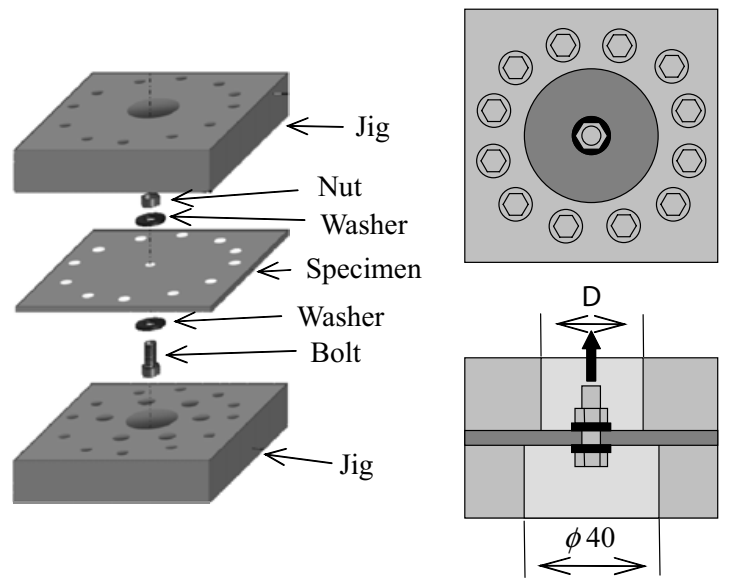

Fig. 2. Assembly of steel plate specimen jointed by a bolt.

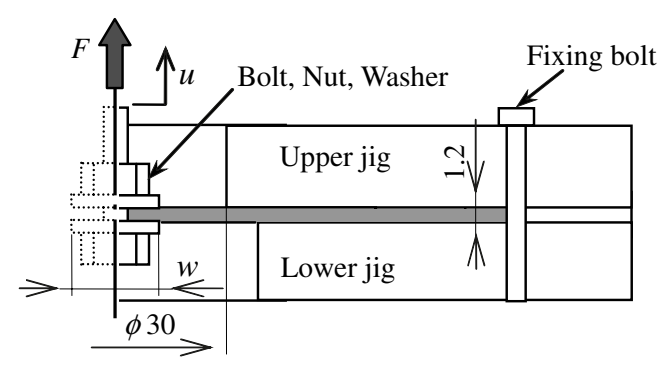

Fig. 3. Schematic of extraction in static test.

$D=30,40,50$, and $80 \mathrm{~mm}$ were employed. However the extractive loads indicated almost the same value in spite of the different of $D$, so that this paper would describe only the case of $D=30 \mathrm{~mm}$.

\subsection{Experimental results in static test}

The extractive static test was performed using a universal testing machine INSTRON 5586 with a cross-head speed of $0.01 \mathrm{~mm} / \mathrm{s}$, as shown in Figure 3.

Figure 4 shows the typical example of the experimental result obtained by static tests: the extractive load $(F)$ versus displacement $(u)$ for the high tensile strength steel plates of $440 \mathrm{MPa}$ class (CR440). Three plates are examined for each washer. It can be seen that the extractive loads increase with increasing of the washer diameter of $w$.

The steel plate specimens after the static extractive test are shown in Figure 5. The specimen plates are extracted out underneath the outer diameter of the washer,

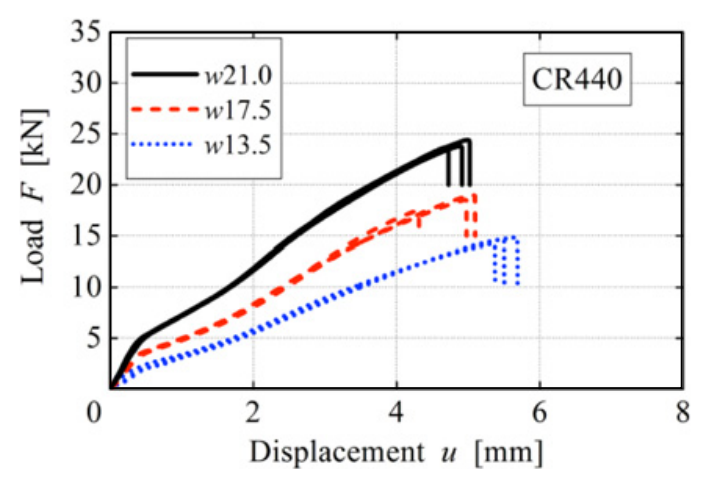

Fig. 4. Extractive load-displacement curves for CR440.

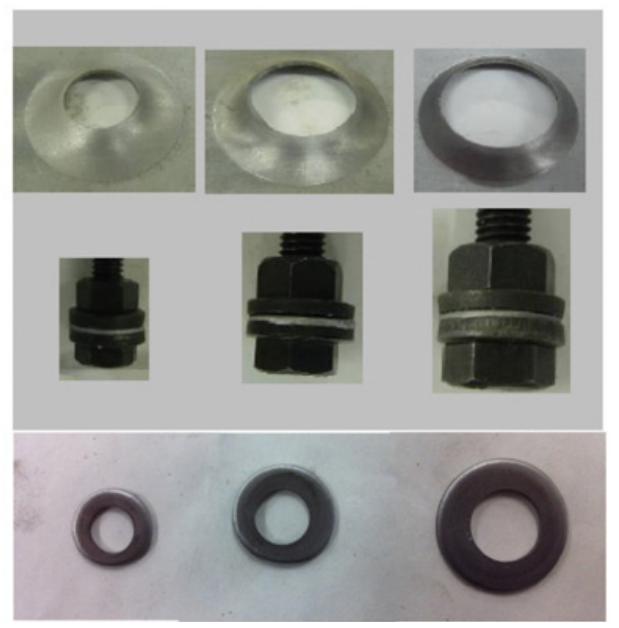

$\begin{array}{lll}\text { (a) } w 13.5 & \text { (b) } w 17.5 & \text { (c) } w 21.0\end{array}$

Fig. 5. Extractive fracture specimens in static tests.

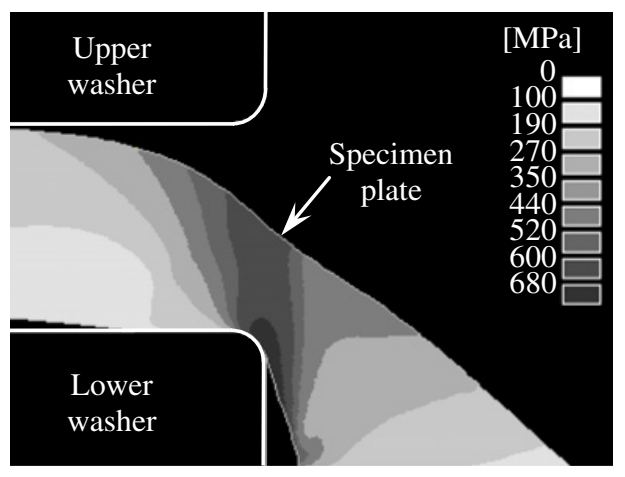

Fig. 6. Distribution of equivalent stress.

respectively. The inside part of specimen plates is caught between washers. Resolving it into a bolt, nuts and washers, as shown in the lowest photograph, a ring is found, respectively.

In order to understand the extractive deformation up to fracture of specimen plates, a numerical simulation was carried out using a FEM code ANSYS. Figure 6 illustrates the distribution of equivalent stress in the vicinity of failure for CR270 and $w=17.5$. The high equivalent stress is observed at the contact region between the specimen plate and the outer edge of a lower washer. Then the specimen 


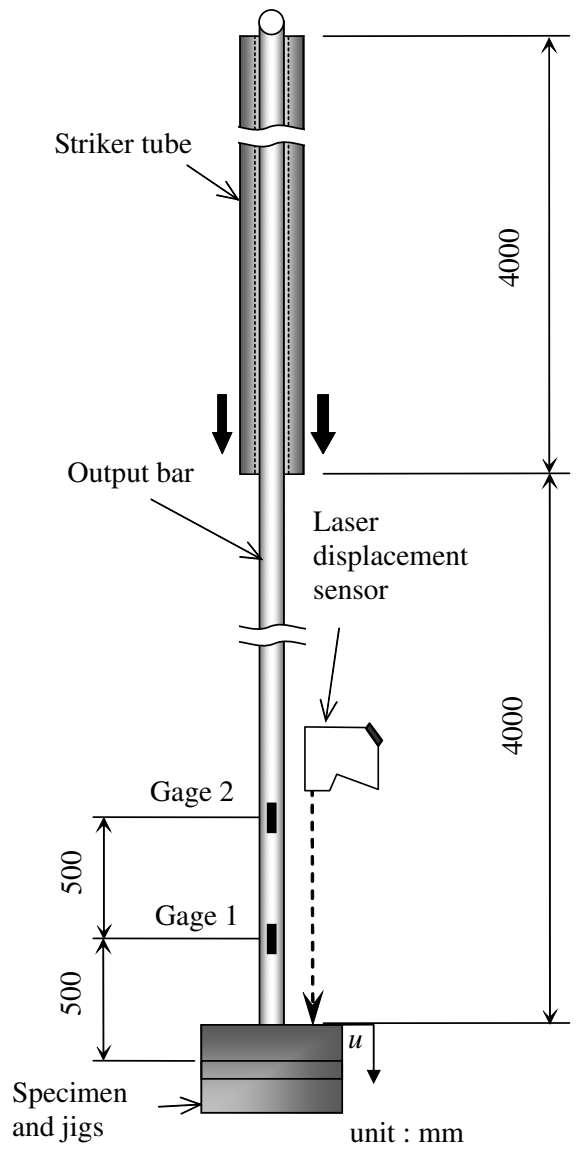

(a) Schematic of impact extractive test.

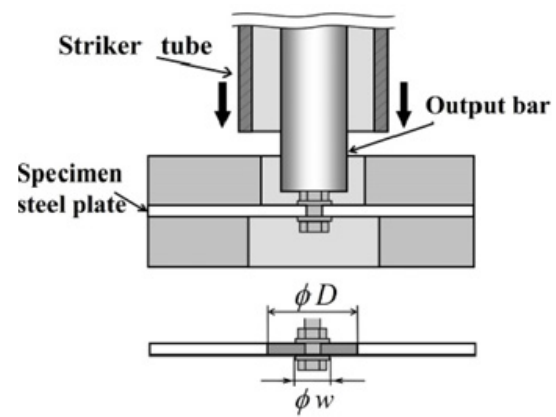

(b) Illustration of impact part.

Fig. 7. (a) Schematic of impact extractive test. (b) Illustration of impact part.

plate seems to break in the direction of out-plane due to the dominant shear stresses.

\section{Impact extractive experiments}

\subsection{Apparatus of impact test and procedure}

Impact extractive tests were performed using a vertical type apparatus of the modified one-bar method for impact test. Figure 7(a) shows the schematic diagram of the set-up. The illustration of the impact part is shown in Figure 7 (b). The output bar made of SUS304 stainless steel has the diameter of $25.0 \mathrm{~mm}$ and the length of

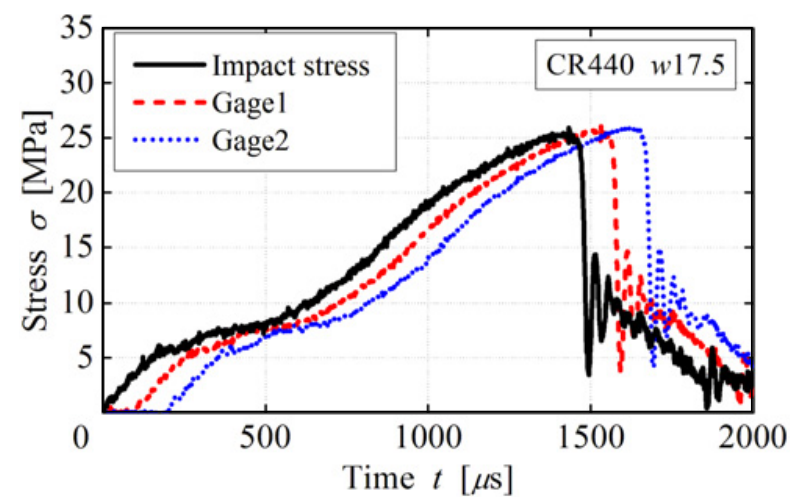

Fig. 8. Impact stress at output bar end.

$8000 \mathrm{~mm}$. The Young's modulus of the bar is $194 \mathrm{GPa}$ and the mass density is $7.76 \times 10^{3} \mathrm{~kg} / \mathrm{m}^{3}$, and then the velocity of elastic wave is $5.02 \times 10^{3} \mathrm{~m} / \mathrm{s}$. The assembly of a specimen plate with a bolt, nuts and jig plates, shown in Figure 2, is fastened at the impact end of the output bar as a flange. The steel striker tube, $42.7 \mathrm{~mm}$ in outside diameter, $35.5 \mathrm{~mm}$ in inside diameter and $4000 \mathrm{~mm}$ in length, was dropped from $4.0 \mathrm{~m}$ in height (the impact velocity is about $V=8.85 \mathrm{~m} / \mathrm{s}$ ). Two semiconductor strain gages (KYOWA, KSP-2-120-E4) are glued at two locations axial-symmetrically to capture stress waves. The signals from the strain gages are passing through bridge boxes and amplifiers (KYOWA, CDV-700A), and recorded by a digital oscilloscope (Nicolet, INTEGRA10) with a sampling time of $1.0 \mu \mathrm{s}$. On the other hand, the variation of the flange displacement $(u)$ with time was measured by a CCD laser displacement sensor (KEYENCE, LK-G155).

\subsection{Experimental results in impact test}

Figure 8 shows the stress waves measured at strain gages 1 and 2 glued on the output bar for the case of the specimen steel plate of CR440 and the washer of diameter $w=$ $17.5 \mathrm{~mm}$. From those waves the variation of the impact stress with time at the output bar end is obtained, and then the impact extractive load $(F)$ can be estimated multiplying it by the cross-sectional area of the output bar.

The flange displacement $(u)$ measured simultaneously by a CCD laser displacement sensor versus time is shown in Figure 9.

Eliminating the time from both of the load-time curves and the displacement-time curves, the diagrams of impact extractive load $(F)$-displacement $(u)$ are obtained. Figure 10 shows the impact extractive load-displacement curves of CR440 for each washer of the diameter $w$. The impact extractive loads increase with increasing of the washer diameter, similarly to those in the static tests. The same results are also obtained on CR270 and CR590 steel plates.

Figure 11 shows the extracted specimen plates of CR440 in the impact test. The fracture figures are almost the same as ones in the static tests. 


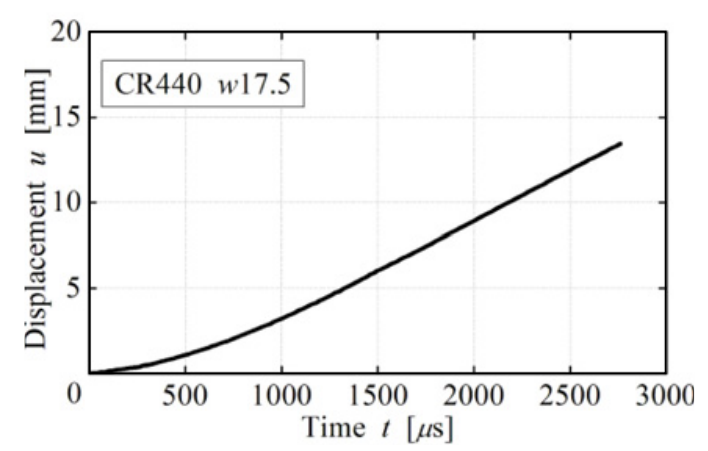

Fig. 9. Displacement-time curve for CR440 and $w=17.5$.

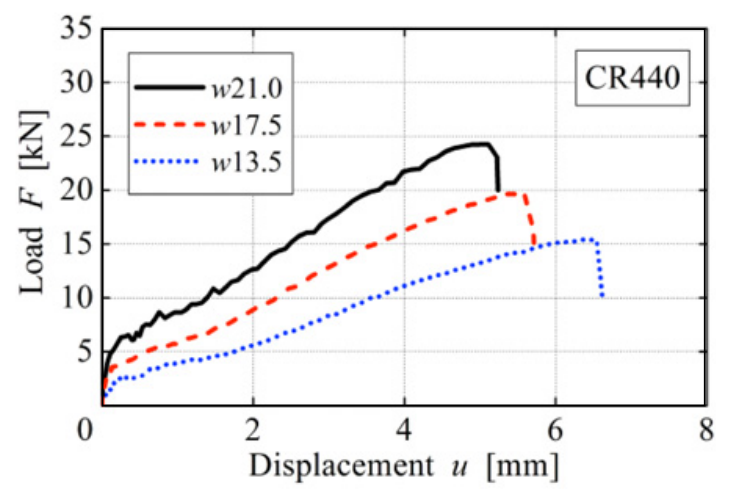

Fig. 10. Impact extractive load-displacement diagram of CR440 under impact velocity $V=8.85 \mathrm{~m} / \mathrm{s}$.

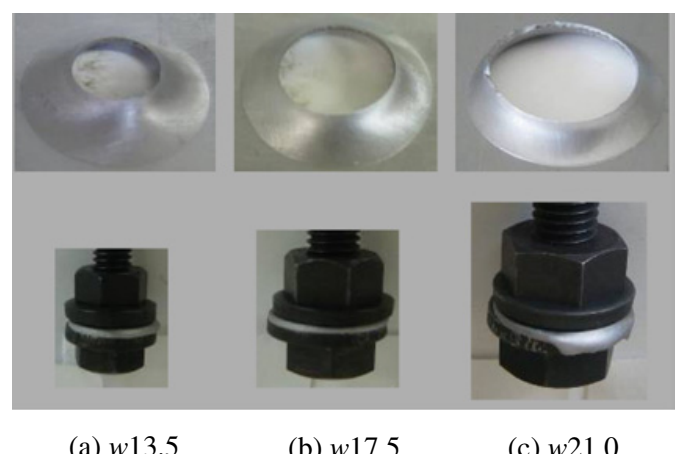
(a) $w 13.5$
(b) $w 17.5$
(c) $w 21.0$

Fig. 11. Extractive fracture specimens in impact tests.

\subsection{Numerical simulations for impact extraction}

Numerical simulations were also carried out using a FEM code LS-DYNA in order to examine the extractive fracture mechanism of jointed steel plates subjected to impact loading. An analytical model used in numerical simulations is illustrated in Figure 12. Two-dimensional, axialsymmetrical analysis was applied. The variation of the displacement $(u)$ with time measured in the experiment was given in numerical calculations. The true stresslogarithmic strain diagram of CR440 is shown in Figure 13. On the basis of this diagram a Material Model 24 setting in the LS-DYNA was employed in the numerical simulation, in which strain rates of materials are taken into consideration.

Figure 14 shows a comparison of the experimental result shown in Figure 8 and a corresponding numerical

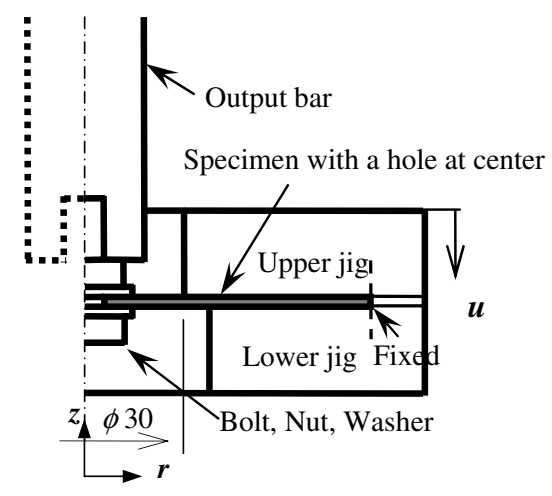

Fig. 12. Analytical model for impact extractive tests.

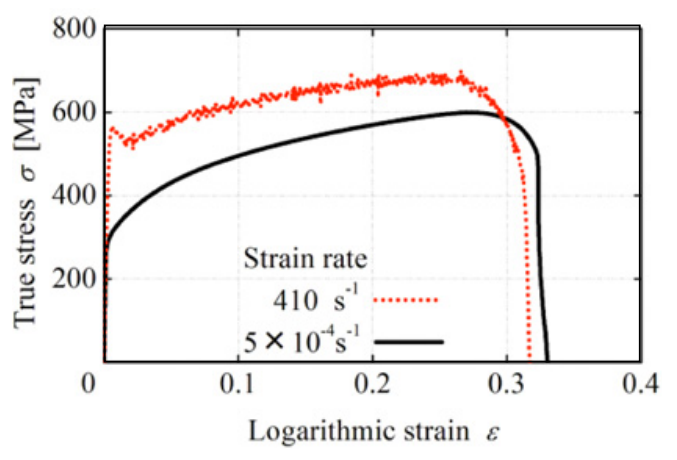

Fig. 13. True stress-logarithmic strain diagram of CR440.

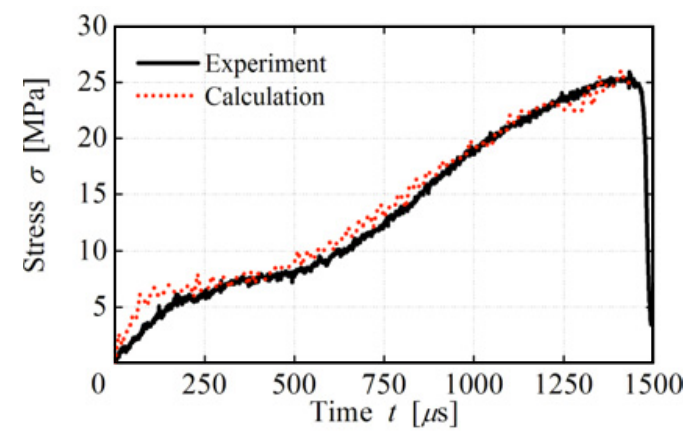

Fig. 14. Comparison of measured and numerical results.

simulation concerning the variation of impact stress with time. The calculated result is approximately agreed with the experimental one.

Figure 15 represents the simulated, sequence distributions of equivalent stress in the cross-sectional area of a steel plate of CR440. The equivalent stress is highly produced around the contact region with the outer edge of a lower washer. Therefore this position is recognized to be the starting one of extractive fracture similar to the static case.

\section{Static and impact extractive stress}

Both the experimental and simulated results may suggest that the shearing stress in the direction of out-plane is dominant to the extractive failure process of jointed steel plates. Figure 16 shows the cross-sectional area of an extracted steel plate. The extractive loads $(F)$ may be 




Fig. 15. Successive distributions of equivalent stress.

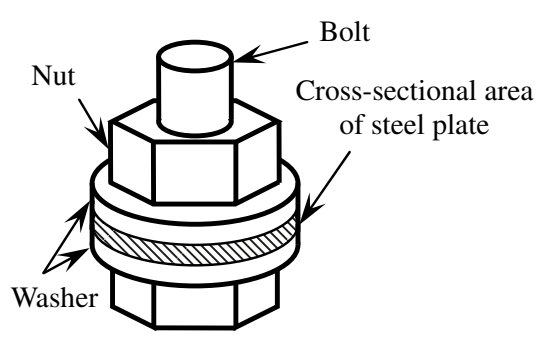

Fig. 16. Extracted cross-sectional area.

normalized dividing by those areas $(A)$. For the sake of convenience, we should call $(F / A)$ the extractive stress hereafter.

Figure 17 shows the relation of the extractive stresses and the displacements for CR440 steel plates in static tests (a), and that in impact tests (b), respectively. The extractive loads increased with increasing of the washer diameter $w$ for both static tests and impact tests, as shown in Figure 4 and Figure 10. However it may be seen that the extractive fracture strength in three curves become similar to each other in spite of the difference of $w$ values. The same results are obtained on CR270 and CR590 steel plates. This fact indicates that a stress-based fracture criterion may be developed for the impact fracture of jointed steel plates of bolted joints used in car bodies.

Figure 18 shows a comparison between the extractive stress-displacement curves in static and impact tests. It can be seen that the impact extractive strengths increase compared with the static ones, and the effect of displacement rate on the strength appears comparatively large in CR270 steel with high rate-sensibility, but does smaller in CR590 steel, because the impact velocity is not so much high in this impact test.

Figure 19 shows the relation of the extractive strength $\sigma_{E}$ and the tensile strength $\sigma_{B}$ for three kinds of the testing steel plates in both static and impact tests. The impact tensile strengths with a strain rate of approximately $340 \mathrm{~s}^{-1}$ were obtained from another impact experiments adopting a split Hopkinson bar [12]. They increase in comparison to the static ones due to the effect of strain rate. The open circles, triangles and squares denote the static results,

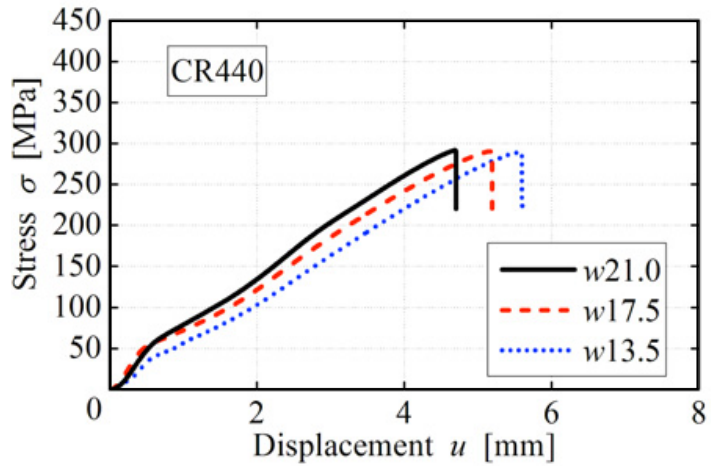

(a) Static extractive stress-displacement diagram.

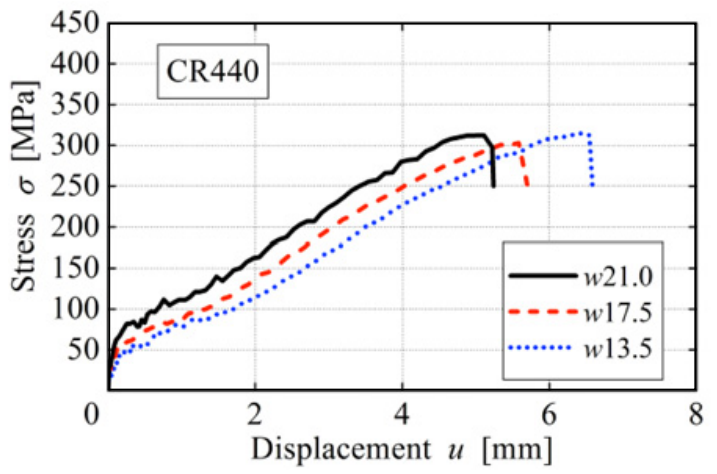

(b) Impact extractive stress-displacement diagram.

Fig. 17. (a) Static extractive stress-displacement diagram. (b) Impact extractive stress-displacement diagram.

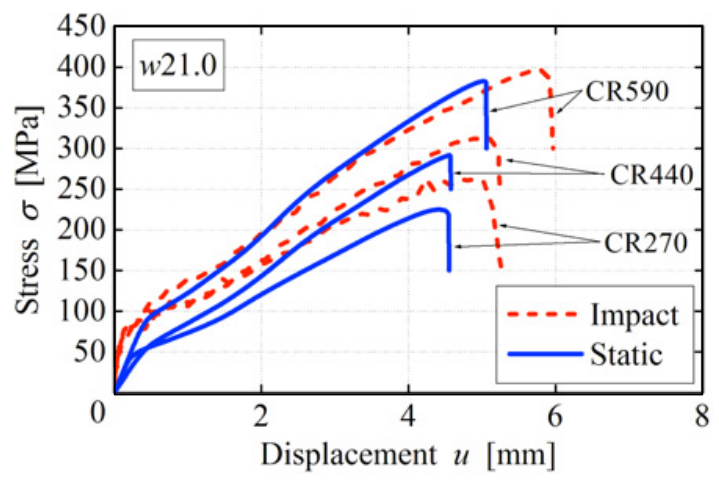

Fig. 18. Comparison of results in static and impact tests.

while the filled ones do the impact results. The impact extractive strengths, which were obtained with an impact velocity of $8.85 \mathrm{~m} / \mathrm{s}$, increase also in comparison to the static ones due to the effect of displacement rate. The plots may lie on a straight line for the static case and the impact case, respectively. The straight lines were fitted by means of the method of least squares. The solid line is for the static results and the broken line for the impact results. This implies that the extractive strength of other steel plates may be estimated only by tensile tests without extractive tests.

\section{Concluding remarks}

The impact behavior and fracture of jointed steel plates used in the suspension pats of cars were examined in 


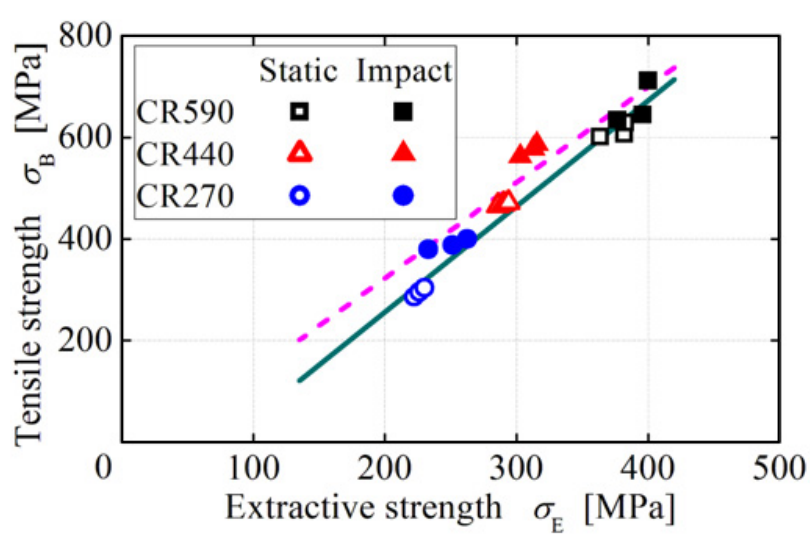

Fig. 19. Relation of tensile strength $\sigma_{\mathrm{B}}$ and extractive strength $\sigma_{\mathrm{E}}$.

experiments and numerical simulations, together with the static characteristics. In this paper attention was given to the extractive deformation and fracture, even though the actual behaviour of bolted joint parts was complicated.

The testing specimens were made of three kinds of steel plates of typical materials for car bodies, i.e., common mild steel of $270 \mathrm{MPa}$, and high tensile strength steel of $440 \mathrm{MPa}$ and $590 \mathrm{MPa}$ class in tensile strength. The impact extraction test of steel plates jointed by a bolt was performed using a modified one-bar method, while the static test was done using a universal testing machine INSTRON 5586. Numerical simulations by a FEM code LS-DYNA were also carried out to compare with the experimental results and to understand the mechanism of extractive fracture process of jointed steel plates.

The impact extractive loads became larger than the static ones due to the effect of displacement rates. The extractive loads increased with increasing of the washer diameter, and the specimen plates were extracted out underneath the outer diameter of the washer for both the static and impact tests. However, the extractive fracture strength, defined as dividing the extractive load by the extracted cross-sectional area, became similar to each other in spite of difference of washer diameters for each steel plate. This suggests that a stress-based fracture criterion may be developed for the impact extractive fracture of jointed steel plates of a bolted joint used in a car body.

\section{References}

1. H. Tsuda, M. Daimaruya, H. Kobayashi, J. Society of Materials Science, Japan, 53, 11(2004), 734-740.

2. H. Tsuda, M. Daimaruya, H. Kobayashi, J. Society of Materials Science, Japan, 54, 7(2005), 921-926.

3. H. Tsuda, M. Daimaruya, H. Kobayashi, J. Society of Materials Science, Japan, 55, 9(2006), 824-830.

4. Y. Takahashi, M. Daimaruya, H. Kobayashi, H. Tsuda, H. Fujiki, Transactions of Japan Society of Mechanical Engineers, A., 73, 732(2007), 891-896.

5. H. Kobayashi, M. Daimaruya, H. Tsuda, K. Horikawa, Y. Yamada, Inter. J. Modern Physics B, 22, 9,10\&11(2008), 1141-1146.

6. A. Yamamoto, Design and Principle of Treaded Fasteners, (Youkendo, 1995).

7. S, Izumi, T. Yokoyama, A. Iwasaki, S. Sakai, Transactions of Japan Society of Mechanical Engineers A., 71, 702(2005), 204-212.

8. M. Daimaruya, H. Fujiki, Y. Uemura, Proceedings of DYMAT 2009 (2009), 867-873.

9. Y. Uemura, Daimaruya, H. Fujiki, H. Kobayashi, K. Nagai, J. Society Materials Science, Japan, 58, 11(2009), 903-909.

10. Y. Uemura, Y. Aihara, M. Daimaruya, H. Fujiki, H. Kobayashi, K. Nagai, J. Society Materials Science, Japan, 60, 7(2009), 662-667.

11. M. Daimaruya, H. Fujiki, H. Shin, H. Kobayashi, K. Nagai, M. Ueda, J. Japanese Society for Strength and Fracture of Materials, 45, 2(2009), 19-27.

12. M. Daimaruya, H. Kobayashi, H. Tsuda, J. Society of Materials Science, Japan, 53, 11(2006), 1240-1246. 